\title{
Operating characteristics of a semiconducting polymer laser pumped by a microchip laser
}

\author{
G. A. Turnbull \\ Organic Semiconductor Centre \& Ultrafast Photonics Collaboration, School of Physics and Astronomy, \\ University of St. Andrews, St. Andrews, Fife, KY16 9SS, United Kingdom \\ P. Andrew and W. L. Barnes \\ Thin Film Photonics Group, School of Physics, University of Exeter, Stocker Road, Exeter, \\ EX4 4QL, United Kingdom \\ I. D. W. Samuel ${ }^{\mathrm{a})}$ \\ Organic Semiconductor Centre \& Ultrafast Photonics Collaboration, School of Physics and Astronomy, \\ University of St. Andrews, St. Andrews, Fife, KY16 9SS, United Kingdom
}

(Received 27 September 2002; accepted 18 November 2002)

We report the demonstration of a compact, all-solid-state polymer laser system featuring a microchip laser as the pump source. The laser was configured as a surface-emitting, two-dimensional distributed feedback laser, based on the conjugated polymer poly(2-methoxy-5( 2 '-ethylhexyloxy)-1,4-phenylene vinylene). Pulsed, band-edge lasing was observed at $636 \mathrm{~nm}$ above a threshold pump energy of $4 \mathrm{~nJ}$. The laser exhibited an energy slope efficiency of $6.8 \%$, with a maximum output energy of $1.12 \mathrm{~nJ}$ at a pump energy of $20.4 \mathrm{~nJ}$. The output beam had an azimuthally polarized annular profile with a beam quality factor $\left(M^{2}\right)$ of 2.2 , close to the theoretical value of the lowest-order Laguerre-Gaussian and Bessel-Gaussian annular modes. We explain the origin of the azimuthal polarization as due to a coherent combination of the resonant fields supported by the two gratings. (C) 2003 American Institute of Physics. [DOI: 10.1063/1.1536249]

In the last few years, semiconducting (conjugated) polymers have emerged as an attractive new gain medium for lasers $^{1-8}$ and optical amplifiers ${ }^{9}$ that are tunable throughout the visible spectrum. These materials exhibit large fluorescence quantum efficiencies, and almost no concentration quenching, allowing them to be used undiluted in the solid state. In such a configuration, the materials are electroluminescent, pointing to the possibility of direct electrical excitation of plastic lasers. The polymers may be readily processed from solution to form various waveguide resonator structures. ${ }^{1,2}$ They are particularly suited for use in onedimensional (1D) and two-dimensional (2D) distributed feedback (DFB) lasers, ${ }^{3-8}$ where the high refractive index contrast of the polymer-substrate interface $(\sim 1.9-1.46)$ can allow strong feedback in very compact resonators.

While there has been a drive to reduce lasing thresholds to allow the use of simple pump lasers and ultimately electrical excitation, to date all semiconducting polymer lasers have been pumped, using expensive, large-frame laser systems (including mode-locked Ti:sapphire, ${ }^{1,3,4,7}$ nitrogen-, and excimer-pumped dye, ${ }^{5-7}$ and flashlamp-pumped $\mathrm{Nd}: \mathrm{YAG}^{2}$ and ruby $^{8}$ lasers). There have also been little quantitative data reported on the output powers of these lasers, with very few published values of output slope efficiency. ${ }^{8}$

In this letter, we demonstrate the use of a microchip laser as the pump source for a surface-emitting DFB polymer laser, thus forming a compact and convenient, all-solid-state laser system with the potential for broad spectral tuning. We present the output energy, efficiency, and spectral character-

\footnotetext{
a) Author to whom correspondence should be addressed; electronic mail: idws@st-and.ac.uk
}

istics of the device, and a detailed analysis of the surfaceemitted laser mode.

The polymer DFB laser comprised an "egg box" corrugated silica substrate covered by a thin film of the conjugated polymer poly(2-methoxy-5-(2'-ethylhexyloxy)-1,4phenylene vinylene) (MEH-PPV). Corrugations on the 25 $\times 25 \times 1 \mathrm{~mm}^{3}$ substrate were initially defined holographically, in two perpendicular directions, in a layer of photoresist. Subsequent chemical development and reactive-ion etching into the silica substrate formed the 2D square array grating structure shown in the atomic force microscope (AFM) image in Fig. 1(a). The grating covered $90 \%$ of the substrate, had a period of $409 \mathrm{~nm}$ in both directions and a peak-to-dip height of $100 \mathrm{~nm}$. The corrugations in the structure provided both distributed laser feedback and a surface-
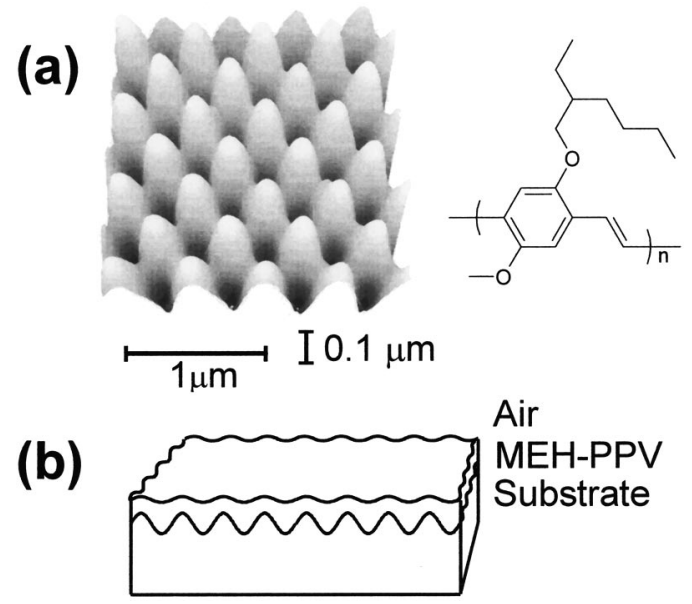

FIG. 1. (a) AFM image (vertical peak-to-dip height=100 nm) of the surface of the silica substrate and chemical structure of MEH-PPV. (b) Waveguide structure of DFB laser. 


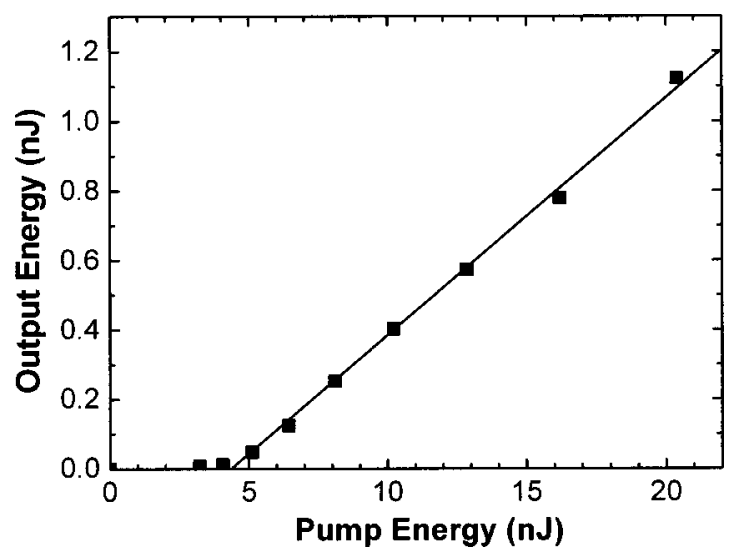

FIG. 2. Output energy from polymer laser as a function of pump energy.

emitted output coupling via second- and first-order Bragg scattering, respectively.

To complete the optical waveguide, a thin film of MEHPPV was spin-coated from solution (5 mg MEH-PPV in $1 \mathrm{ml}$ chlorobenzene; spin speed of $1200 \mathrm{rpm}$ ) onto the silica, forming a polymer layer with an average thickness of $\sim 100$ $\mathrm{nm}$. As a result of the deposition process, the top surface of the polymer layer was substantially more planar than the silica substrate. The silica-polymer-air structure [Fig. 1(b)] formed an asymmetric slab waveguide that supported only the fundamental transverse electric mode within the emission band of the polymer.

The polymer laser was mounted in a vacuum chamber and held under a vacuum of $10^{-4}$ mbar to isolate it from oxygen and water. It was optically pumped by a frequencydoubled, passively $Q$-switched $\mathrm{Nd}: \mathrm{YVO}_{4}$ microchip laser (Alphalas $\mathrm{GmbH}$ ) which produced $\sim 1 \mathrm{~ns}$ pulses at $532 \mathrm{~nm}$ with a repetition rate of $5 \mathrm{kHz}$. The dimensions of the microchip laser were $40 \times 40 \times 60 \mathrm{~mm}^{3}$ leading to a very compact system. The output of the pump laser was attenuated using neutral density filters, and then focused using a $63-\mathrm{mm}$ focal length, spherical lens to a spot of half-width of $100 \mu \mathrm{m}$ (horizontal) by $80 \mu \mathrm{m}$ (vertical) on the surface of the polymer. The pump beam was incident at an angle of $\sim 20^{\circ}$ to the waveguide normal; alignment of the pump laser was trivial, with side-pumped lasing possible across the full area of the corrugations.

The spectral output from the polymer laser was measured around normal incidence using a fiber-coupled CCD spectrometer of $\sim 0.7 \mathrm{~nm}$ resolution. Output energies from the laser were measured using a calibrated silicon energy meter for a range of excitation powers. The beam profile and divergence of the laser output were measured using a CCD beamview analyzer (Coherent Inc.).

When excited above threshold, the polymer laser emitted a bidirectional output beam along the waveguide normal. The output pulse energies emitted into the silica- and aircladding layers were approximately equal. Figure 2 shows the dependence of the total emitted output energy on the absorbed pump energy. The laser had an oscillation threshold of $4 \mathrm{~nJ}$, above which the output pulse energy increased linearly. The laser had an energy slope efficiency of $6.8 \%$ (for comparison, the photoluminescence quantum efficiency of MEH-PPV studied here was $25 \%^{10}$ ) with an output of 1.12 $\mathrm{nJ}$ at $636 \mathrm{~nm}$ for a pump energy of $20.4 \mathrm{~nJ}$. This slope effi-

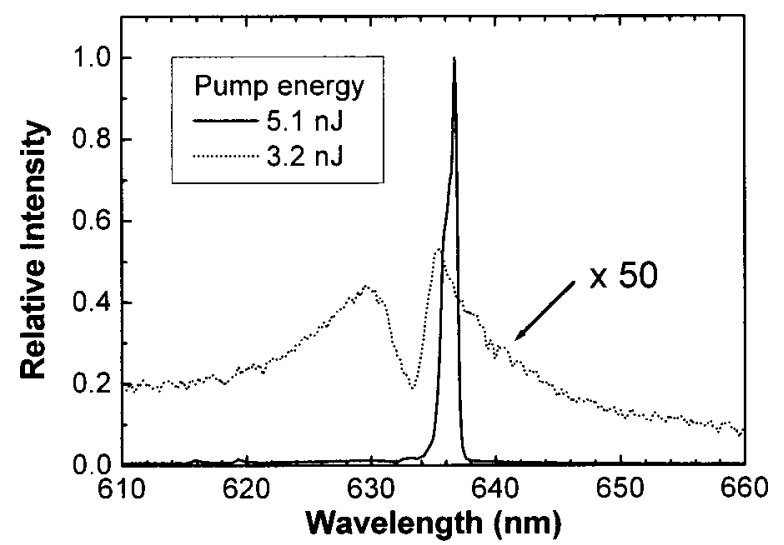

FIG. 3. Emission spectra normal to the waveguide plane for pump pulse energies of 3.2 (dotted line) and $5.1 \mathrm{~nJ}$ (solid line).

ciency is approximately twice that reported for a $1 \mathrm{D}$ DFB laser based on thianthrene-diocytyloxy-PPV. ${ }^{8}$

The spectral characteristics of the laser, measured along the waveguide normal, are shown in Fig. 3. The dotted curve represents the emission spectrum when operating just below laser threshold, at a pump energy of $3.2 \mathrm{~nJ}$. This spectrum shows two peaks, separated by a narrow dip at $633 \mathrm{~nm}$. The dip corresponds to a photonic stopband, resulting from the distributed feedback of waveguide modes by the 2D corrugations. Above laser threshold, (solid line in Fig. 3) the emission spectrum was dominated by a narrow peak at $636 \mathrm{~nm}$, of $\sim 1 \mathrm{~nm}$ in linewidth. This peak arose on the edge of the photonic stopband, where the density of photon states is highest. Lasing occurred only on one side of the stopband because of a substantial difference in output coupling losses of the modes at each band edge. ${ }^{6,11}$ The standing wave field at the long-wavelength band edge is poorly coupled to radiation, and so has a lower lasing threshold than the shortwavelength edge. The 636-nm wavelength of this laser was determined by the current choice of DFB period. Using a range of DFB gratings, we have previously tuned the lasing wavelength in MEH-PPV through a range of 612 to $632 \mathrm{~nm} .^{5}$

The far-field transverse mode of the laser beam was found to be annular in profile, as is shown in the center of Fig. 4. The mode was generally symmetrical about the two grating axes, although the exact profile of the beam varied slightly as the pump beam was translated across the polymer laser. The four surrounding beam profiles in Fig. 4 show the same laser mode when measured through a linear polarizer, aligned to pass electric fields parallel to the double-headed arrows. The polarized profiles take the shape of a TEM 10 mode that rotates with the direction of the polarizer. It is clear from this data that the emitted laser beam was azimuthally polarized.

The azimuthal polarization may be understood by considering the horizontal and vertical polarized components of the laser mode (Fig. 4), which are polarized parallel to the axes of the two gratings in the structure. These two components resemble (albeit with lower divergence) the polarized, double-lobed emission observed from 1D surface-emitting DFB lasers. ${ }^{12}$ This double-lobed emission is a characteristic of the antisymmetric band edge mode that is weakly coupled to radiation. ${ }^{11,12}$ In the 1D DFB laser, the radiation coupling is weakest in the center of the resonator, where the counterAIP license or copyright; see http://apl.aip.org/apl/copyright.jsp 


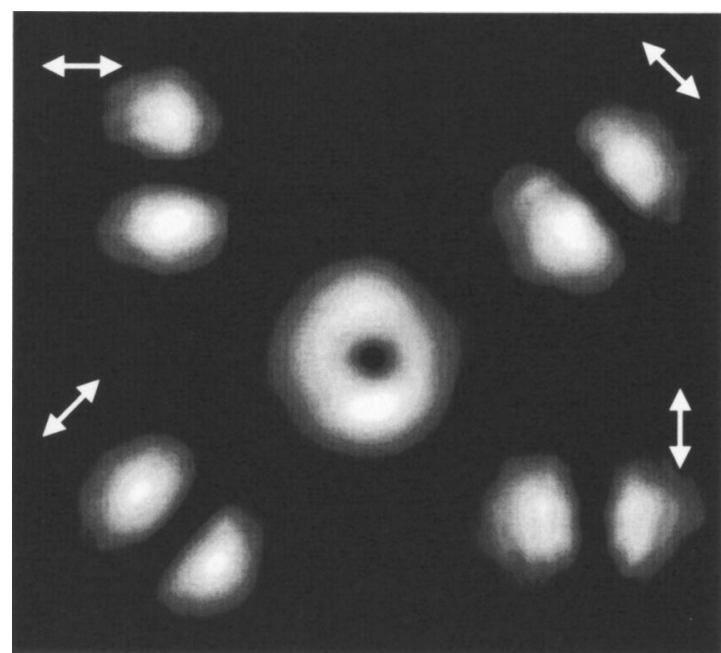

FIG. 4. Central profile: transverse mode of the polymer laser beam. Surrounding profiles: transverse laser mode when measured through a linear polarizer, aligned parallel to the double-headed arrows.

propagating band-edge modes have equal amplitudes ${ }^{11}$ and interfere to produce a standing wave with nodes at the peaks and troughs of the grating. ${ }^{13}$ At the edges of the pumped region, the counter-propagating modes have unequal amplitudes, reducing the contrast of the interference pattern. The fields in these regions are more efficiently coupled to radiation, leading to the double-lobed emission pattern. The two lobes of the emitted mode oscillate in antiphase, which means that the electric field pattern has a twofold rotational symmetry. In our bigrating structure, there is one such field component for each orthogonal grating; these couple together with a phase that leads to an azimuthal polarization. We can therefore identify the azimuthally polarized mode as due to a coherent combination of the resonant fields supported by the two gratings.

To quantify the quality of the laser beam, we measured the far-field divergence and $M^{2}$ beam quality factor. ${ }^{14}$ The divergence of the beam is shown in the inset of Fig. 5. Beamwidths were calculated by integrating the intensity profiles along axes parallel to each grating (nominally referred to below as horizontal and vertical axes), and measuring the $1 / e^{2}$ width. The beamwidth was larger in the vertical direction than the horizontal, with half-angle divergences $\theta$ of $6.3 \pm 0.7$ and $5.2 \pm 0.1 \mathrm{mrad}$, respectively.

To measure the $M^{2}$ value of the beam, we next focused the polymer laser beam to a spot of $\sim 50-\mu \mathrm{m}$ radius using a 50-mm focal length lens, and measured the variation in beamwidth with axial position through the focus. The results of the measurements in the horizontal and vertical directions are shown as squares and circles in Fig. 5. The displaced foci of the two data sets show that the laser beam was astigmatic. The curves in the figure are theoretical fits of the beam radius $w=w_{0} \sqrt{1+\left(M^{2} \lambda z / \pi w_{0}^{2}\right)^{2}}$, where $w_{0}$ is the waist radius, $\lambda$ the laser wavelength, and $z$ the axial distance from the beam waist. The theoretical fits gave a $M^{2}=2.1$ in the horizontal direction, and a $M^{2}=2.3$ in the vertical; the geometric average of these is $M^{2}=2.2$. This value is comparable with other low-order annular modes; both the Laguerre-Gaussian $\mathrm{LG}_{0}^{1}$ mode,${ }^{14}$ and Bessel-Gaussian beam of order $1,{ }^{15}$ have a theoretical $M^{2}=2$. Finally, we can use the $M^{2}$ values to calculate the size of the beam at the surface of the polymer laser.

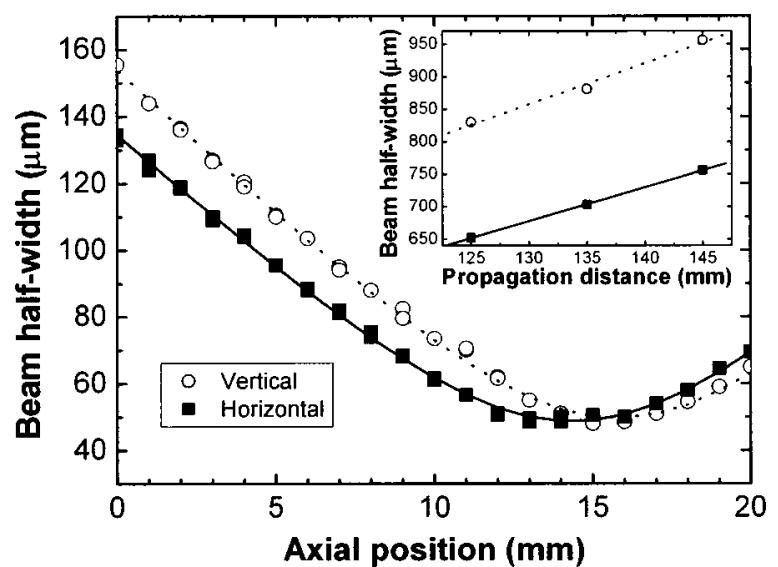

FIG. 5. Horizontal and vertical half-widths of the focused polymer laser beam. Inset shows the change in beam half-widths of the emitted laser beam with propagation distance from the polymer waveguide.

Assuming that the output beam has a waist located at the surface of the laser, the half-width of the beam is given by $w_{0}=M^{2} \lambda / \pi \theta$. This yields horizontal and vertical halfwidths of 82 and $74 \mu \mathrm{m}$, respectively, which are similar to the dimensions of the pump excitation.

In summary, we have demonstrated a compact solid-state laser system based on a microchip laser pumped polymer laser. The laser exhibited a low threshold energy of $4 \mathrm{~nJ}$ and a slope efficiency of $6.8 \%$. The output beam had an azimuthally polarized annular profile, which we attribute to a coherent combination of the resonant fields supported by the two gratings. The beam had an $M^{2}$ of 2.2, close to the theoretical value of the lowest-order Laguerre-Gaussian and BesselGaussian modes. We conclude that semiconducting polymer lasers are well suited as compact and efficient sources that can greatly extend the spectral range of microchip lasers.

We are grateful to EPSRC for financial support, and Covion for the supply of the polymer. One of the authors (I.D.W.S.) is a Royal Society University Research Fellow; another (G.A.T.) is an EPSRC Advanced Research Fellow.

${ }^{1}$ N. Tessler, Adv. Mater. 11, 363 (1999).

${ }^{2}$ M. D. McGehee and A. J. Heeger, Adv. Mater. 12, 1655 (2000).

${ }^{3}$ C. Kallinger, M. Hilmer, A. Haugeneder, M. Perner, W. Spirkl, U. Lemmer, J. Feldmann, U. Scherf, K. Mullen, A. Gombert, and V. Wittwer, Adv. Mater. 10, 920 (1998).

${ }^{4}$ S. Riechel, C. Kallinger, U. Lemmer, J. Feldmann, K. Gombert, V. Wittwer, and U. Scherf, Appl. Phys. Lett. 77, 2310 (2000).

${ }^{5}$ G. A. Turnbull, T. F. Krauss, W. L. Barnes, and I. D. W. Samuel, Synth. Met. 121, 1759 (2001).

${ }^{6}$ G. A. Turnbull, P. Andrew, W. L. Barnes, and I. D. W. Samuel, Phys. Rev. B 64, 125122 (2001).

${ }^{7}$ C. Bauer, H. Giessen, B. Schnabel, E.-B. Kley, C. Schmitt, U. Scherf, and R. F. Mahrt, Adv. Mater. 13, 1161 (2001).

${ }^{8}$ W. Holzer, A. Penzkofer, T. Pertsch, N. Danz, A. Brauer, E. B. Kley, H. Tillmann, C. Bader, and H.-H. Horhold, Appl. Phys. B: Lasers Opt. 74, 333 (2002).

${ }^{9}$ J. R. Lawrence, G. A. Turnbull, and I. D. W. Samuel, Appl. Phys. Lett. 80, 3036 (2002).

${ }^{10}$ J. A. E. Wasey, A. Safonov, I. D. W. Samuel, and W. L. Barnes, Phys. Rev. B 64, 205201 (2001).

${ }^{11}$ R. F. Kazarinov and C. H. Henry, IEEE J. Quantum Electron. 21, 144 (1985).

${ }^{12}$ S. H. Macomber, J. S. Mott, R. J. Noll, G. M. Gallatin, E. J. Gratrix, S. L. O’Dwyer, and S. A. Lambert, Appl. Phys. Lett. 51, 472 (1987).

${ }^{13}$ P. Andrew and W. L. Barnes (unpublished).

${ }^{14}$ M. W. Sasnett, in The Physics and Technology of Laser Resonators, edited by D. R. Hall and P. E. Jackson (Hilger, Bristol, 1989) p. 132.

${ }^{15}$ R. Borghi and M. Santarsiero, Opt. Lett. 22, 262 (1997). 